\title{
UNA METODOLOGÍA INTERPRETATIVA PARA EL ESTUDIO DE LOS MOVIMIENTOS SOCIALES. ENMARCAMIENTOS Y CULTURA. UNA VISIÓN DESDE MÉXICO
}

\author{
Luis Fernando Villafuerte Valdés ${ }^{1}$ \\ Universidad Veracruzana, México \\ fvillafuertev@hotmail.com
}

Recepción: 30/05/2008

Evaluación 01/06-15/09/2008

Aceptación: 29/09/2008

Artículo de Reflexión

\section{RESUMEN}

La relación entre sistema político, intelectuales y el debate académico tuvo un vínculo muy estrecho en México, tanto que determinaba el debate sobre las teorías "hegemónicas" a emplear en el ambiente académico del país, por lo que cuando se agota la vigencia de los referentes políticos autoritarios, se modifican las relaciones académicas y se abre la posibilidad de incorporar nuevas teorías para explicar lo social.

Palabras Claves: Autoritarismo, movimientos sociales, ideología, cultura, democracia.

\footnotetext{
${ }^{1}$ Doctor en Ciencias Políticas y Administración Pública. Profesor investigador de la Facultad de Ciencias Administrativas y Sociales Universidad Veracruzana, México.
} 


\title{
AN INTERPRETIVE METHODOLOGY FOR THE STUDY OF THE SOCIAL MOVEMENTS. BOUNDERIES AND CULTURE. A VISION FROM MEXICO
}

\author{
Luis Fernando Villafuerte Valdés \\ Universidad Veracruzana, México \\ fvillafuertev@hotmail.com
}

\begin{abstract}
The relation among political and intellectual system and the academic debate had a very narrow link in Mexico, so much that it was determining the debate on the "hegemonic" theories to be used in the academic environment of the country. This is why when the force of the political authoritarian models becomes exhausted, the academic relations are modified and there is the open possibility of incorporating new theories to explain the social process.
\end{abstract}

Key Word: Authoritarism, social movements, ideology, culture, democracy.

\section{INTRODUCCIÓN}

La conformación del sistema político mexicano se construyó bajo parámetros que fusionaron los aspectos institucionales con los sociales, ya que se creó un régimen político en donde todos los sectores sociales del país fueron beneficiados bajo los esquemas de las políticas sociales, así: la seguridad social, la creación de un sistema educativo básico apuntalado por el sistema de educación superior pública, las políticas de ayuda a sectores marginados de la población, etcétera, lograron incorporar a toda la población dentro de la vida revolucionaria institucionalizada, edificada y garantizada por el Estado mexicano.

La capacidad regenerativa del sistema se basó en su capacidad de impulsar a las clases medias a través de un sistema educativo de nivel superior que privilegiaba el ascenso social como dispositivo de control social y no como un mecanismo que permitiera desaparecer las desigualdades sociales generadas por un modelo económico centralizador y selectivo a la hora de repartir sus beneficios. Las clases medias jugaron un proceso dual en esta época de conformación del sistema político en México, por un lado, (a) fueron las principales heredadas de las políticas estatales entre los años 1940 a 1960, donde a partir del esquema educativo se permitió la movilidad y el ascenso social de grandes contingentes de la población, generándose un crecimiento urbano muy evidente, y (b) estás 
fueron educadas bajo la ideología del nacionalismo revolucionario por lo que no cumplieron un papel crítico en relación a las injusticias distributivas del sistema político, ya que fueron desmovilizadas desde diferentes políticas bien definidas. Así, las condiciones de estabilidad del país fueron una distribución de los beneficios económicos, un discurso nacionalista apoyado en estas clases medias y puestos de trabajo en el aparato burocrático del Estado.

Estas características se hicieron extensivas a los sectores académicos en el país, en donde los enfoques utilizados para el análisis social partían de dos lógicas, las cuales eran: (a) un proceso de sobreideologización, -básicamente centrado en el marxismo-, o en su defecto, (b) perspectivas que legitimaban al sistema político. Sin embargo, a pesar de esta tradición, se fue gestando un proceso gradual de crítica desde algunos sectores de clase media a partir de la segunda mitad del siglo XX. Factores externos como la guerra fría, el ascenso del discurso socialista y posteriormente las revoluciones socialistas en países de América latina a nivel internacional y transformaciones a nivel interno como una educación superior que empezó a influirse con la educación socialista, así como la aparición de centros clandestinos de educación para obreros, profesores exiliados de sus países de origen por sus ideas políticas, radicalizan a sectores de las clases medias, sobre todo después de los años sesenta, teniendo su máxima expresión en el movimiento del 68, en el cual jóvenes universitarios hijos de las clases medias de los años cuarenta protestan contra el autoritarismo del Estado, dándose la semilla del posterior nacimiento de la sociedad civil en México.

Así, este ensayo tiene la finalidad de hacer una reconstrucción del ambiente político social en donde se insertan las visiones y las teorías que estudiaban a los movimientos sociales en México, tanto desde los años del autoritarismo político social y académico, hasta los momentos en que la lógica autoritaria deja de tener sentido debido a las reformas económicas neoliberales impuestas a finales de los años ochenta del siglo pasado.

Este artículo cuenta con tres apartados, (a) un apartado historiográfico, el cual reconstruye el entorno y el ambiente de la situación académica del estudio de los movimientos sociales en su relación al sistema político mexicano, (b) un segundo apartado que trata las transformaciones sociales producto de la aplicación de las medidas neoliberales en México y su subsecuente influencia en las formas de movilización social, lo que trae como consecuencia, la utilización de nuevas teorías para explicar a los movimientos sociales y por último, (c) se hará una revisión exhaustiva de los modelos teóricos de los enfoques cognitivos de los movimientos sociales, así como el desarrollo de la estrategia metodológica de los enmarcamientos, que son producto de esta perspectiva teórica. Para concluir con una serie de reflexiones acerca del potencial de análisis que conlleva el desarrollo y la aplicación de estas teorías cognitivas y la metodología de los 
enmarcamientos para la explicación de los fenómenos de organización social en México y en general en el resto de América Latina.

\section{El estado del estudio de los movimientos sociales en México. De las clases sociales a la lucha democrática}

El estudio de los movimientos sociales ha presentado diversas teorías que abordan el objeto de estudio desde diversos ángulos del problema, los cuales van desde las teorías más estructuralistas, que visualizan el problema de los movimientos sociales como producto de un desajuste en los procesos de asignación de los mecanismos de reproducción material y simbólica de la población, hasta las escuelas que analizan el problema a partir de la generación de nuevos referentes culturales e identitarios.

El estado de la investigación sobre los movimientos sociales en América latina, tuvo la característica que estuvo fuertemente influenciado por el contexto político en el continente, lo que privilegio ciertas escuelas y teorías en detrimento de otras, así, las escuelas de corte más estructuralista como la de Movilización de Recursos o la de la Estructura de Oportunidades Políticas no tuvieron un gran auge en los estudios empíricos acerca del tema, y en su lugar, los enfoques marxistas cubrieron el espectro analítico del tema durante los años mil novecientos sesenta hasta aproximadamente los años mil novecientos ochenta.

Esto originó que en América latina, y específicamente en México, el estudio de los movimientos sociales se caracterizara por un análisis fuertemente sesgado a lo ideológico, y se visualizarán como un todo unificado y sin distinciones entre ellos.

Esto se debió a que en los años mil novecientos sesenta a los ochenta, había una gran influencia en el ambiente intelectual de México por el triunfo de la revolución cubana, por lo tanto los proyectos de cambio político estaban conformados por un maximalismo en el sentido de la destrucción del Estado. La percepción de la participación social estaba permeada por el hecho de que los "únicos movimientos sociales considerados dignos de ese nombre fueron los populares, clasistas y básicamente aquellos que reclamaron su autonomía frente al estado". ${ }^{2}$ El hecho de que a estos movimientos se les tratara de manera homogénea se debió a la fuerte tradición marxista dogmática de esos años, además que no existía una diferenciación en cuanto a las estrategias, reclamos y el referente de a quién dirigir las demandas. ${ }^{3}$

\footnotetext{
2 OLVERA RIVERA, Alberto J. (1999): Movimientos Sociales. Desafíos Teóricos y

Metodológicos. México. Ediciones de la Universidad de Guadalajara. p. 112.

${ }^{3}$ Que en este caso era el Estado, ya que era el único referente para el impulso del proyecto nacional.
} 
Esto se generó debido a que el sistema político que emanó de la revolución Mexicana tuvo como característica la fusión entre el Estado, el mercado, la economía y los actores políticos, lo que trajo como consecuencia un esquema de control social muy vertical, cohesionado a través del corporativismo y una ausencia real de un espacio público crítico y autónomo en cuanto a su vinculación con el poder político, ${ }^{4}$ por lo que se privilegió una sobrepolitización de las relaciones sociales con la consecuencia que todas las formas de asociación tanto públicas como privadas estuvieran mediadas, controladas y fiscalizadas por el Estado. ${ }^{5}$

En este sentido, el régimen político mexicano manejó un discurso muy ligado a los derechos sociales aunque estos no se respetarán, ya que se asoció los derechos sociales con los económicos, de tal forma que dentro del imaginario social de México se vinculó la idea de desarrollo económico como el eje principal de la vida política, sin importar los derechos políticos o sociales, lo que trajo como consecuencia la presencia de fraudes políticos legitimados socialmente siempre y cuando hubiera beneficios económicos para al población. El peso de esta visión social sigue imperando en México y el resto de América latina en este primer lustro del siglo XXI, ya que en los últimos datos del latinobarómetro, una gran parte de la población está dispuesta a vivir bajo un régimen militar o dictatorial, siempre y cuando éste les garantice desarrollo económico y material. ${ }^{6}$

A partir de lo anterior, toda movilización social en México tenía que utilizar un discurso más agresivo en este tema, lo que permitió que muchos de los movimientos sociales coincidieran en posiciones radicales de izquierda y por lo tanto, fueran cooptados por ellos.

Estos movimientos manejaban un discurso muy anclado en las clases sociales, por lo que su tipificación podría ser catalogada en términos de Offe como un "viejo movimiento social o "viejo paradigma". Este ambiente originó que a pesar de que pudiera haber identidades "emergentes" dentro de estas movilizaciones, éstas no eran estudiadas debido al tipo de análisis que se empleaba en el país (la visión marxista de la destrucción o transformación total del sistema político).

Así, la introducción del corporativismo en todos los aspectos de la vida cotidiana originó que no se diferenciaran los distintos niveles de la estructura social, dando como resultado, por un lado, una movilización social funcional

\footnotetext{
${ }^{4}$ La estructura corporativa se construyó de tal forma que no sólo se controlaba al obrero en su aspecto laboral sino que se le implicaba en actos de corrupción o de irregularidades laborales, de tal manera que el obrero, campesino o burócrata quedara sujeto a las redes de "favores" que se había construido para el control total de las bases productivas, no sólo en su centro laboral sino también en su espacio privado, familiar y público. Para más información de este tema ver a Paz (1990). ${ }^{5}$ ARCHIVO HISTÓCIO MUNICIPAL DE CAZONES DE HERRERA.

${ }^{6}$ BIBLIOTECA VERACRUZANA, Colección de Publicaciones Periodicas.
} 
condenada a ser cooptada o a legitimar a la larga las acciones del Estado y, por otro lado, una percepción de la acción política referida solamente a los aspectos institucionales, olvidando los aspectos simbólicos o culturales de los procesos de cambio político; así, los movimientos limitaban sus acciones, sus demandas y sus alcances, por lo que estaban irremediablemente obligados a toparse con toda la fuerza del Estado. Su autolimitación para conseguir bases sociales y su "inevitable corporativización” o represión era cuestión de tiempo.

En este sentido, tendríamos tres características que definirían la situación del estudio de los movimientos sociales en estos años: (a) sólo se asumía como movimientos sociales aquel movimiento con un trasfondo revolucionario, (b) se ratificó a los mismos debido a que se les homogenizó y su estudio se ubicó sólo en analizar los discursos de los líderes, así como sus acciones de protesta y (c) se ignoró por completo movimientos urbanos de clases medias o empresariales, ya que fueron tachados de reaccionarios o pro sistémicos, sin embargo éstos tuvieron un papel fundamental en el proceso de cambio político del país tanto en lo electoral, como en la lucha por los derechos civiles.

Por otro lado, en esos mismos años, en el ambiente urbano, se estaba generando una serie de transformaciones culturales muy importantes, ${ }^{8}$ sobre todo entre algunos sectores de las clases medias y los empresarios, cambios que los intelectuales y los partidos políticos no pudieron anticipar, ya que su reduccionismo teórico tenía como característica que sus herramientas conceptuales estaban encaminadas a explicar la confrontación total de los movimientos sociales contra el Estado, y buscar o ver a la clase obrera o "el sujeto revolucionario" como el único actor válido o legítimo para construir movimientos sociales.

Para los primeros años de la década de los ochenta del siglo XX se empiezan a dar las primeras movilizaciones en defensa del voto. Estas primeras movilizaciones van a evidenciar el cambio de referentes para la acción social colectiva; ahora las demandas tenían un carácter cultural, y tenían como eje el de la solidaridad y la identidad demostrada en la movilización civil en el año 1985 después de los terremotos de la ciudad de México. El desbordamiento civil inauguró una nueva etapa en la conceptualización de los movimientos sociales. ${ }^{9}$

7 OLVERA RIVERA, Alberto J. (1999): Movimientos Sociales. Desafíos Teóricos y

Metodológicos. México. Ediciones de la Universidad de Guadalajara. pp. 119-120.

${ }^{8}$ Transformaciones de carácter protodemocrático o predemocrático.

${ }^{9}$ EL DIARIO DE XALAPA, Diario de la Provincia, 20 de enero de 2007. 


\section{La lucha por la democratización y la movilización social identitaria}

Los intentos de autonomía de los movimientos sociales durante los años cuarenta a ochenta del siglo pasado en México terminaron en su mayoría de manera muy dramática; ${ }^{10}$ sin embargo, las experiencias de principios de los años ochenta, demostró que la manera en que se podía consolidar un movimiento social era buscando su autonomía frente al Estado, no sólo en términos de su conformación, sino también en sus demandas, es decir, era necesario rescatar el aspecto cultural o simbólico de la lucha, de tal manera que no tuvieran que pasar por las instancias institucionales y así evitar la posible corporativización por parte del poder estatal.

Se evidenció que los movimientos sociales no eran homogéneos ni su naturaleza estaba establecida sólo por determinaciones estructurales; estos cambios fueron impulsados por los sectores de clase media y empresarios, sectores a los que no se les tomó en cuenta como posibles agentes de cambio político - México.

Sin embargo, la efervescencia de la movilización ciudadana se puede ubicar en el año de 1988 con el fenómeno político que significó el Frente Democrático Nacional encabezado por Cuauhtémoc Cárdenas, quien enarboló la idea del fin del régimen político prísta a partir de la resistencia civil. La izquierda confió sobremanera en la acción electoral, por lo que la apuesta a los procesos electorales como eje del cambio político fue evidente, esto redujo una vez más la concepción de los movimientos sociales, a grupos cautivos y clientelas políticas que podían ser utilizadas de manera instrumental, los intelectuales en México ubicaron a los estudios electorales así como a la ingeniería electoral como ejes fundamentales del proceso de transición política.

Los factores que determinaron el agotamiento del estudio de la acción colectiva desde la perspectiva de los movimientos sociales populares se ubican dos vertientes: por un lado, (a) el impacto de las medidas neoliberales dentro del tejido social de México y (b) los mecanismos emergentes de cooptación social bajo el eje del neocorporativismo gubernamental.

El primer inciso se refiere a cómo las viejas identidades políticas se pierden ante el embate del ajuste económico, los campesinos, los obreros o los grupos

\footnotetext{
${ }^{10}$ Las movilizaciones de ferrocarrileros, maestros y médicos en 1956 terminaron con el uso de la represión y el encarcelamiento de los líderes, la misma suerte corrieron los estudiantes en 1968, los intentos de organizar asociaciones obreras independientes terminaron cooptadas bajo los líderes corruptos (conocidos también como líderes charros), por lo que nunca se consolidó un sindicalismo independiente. Solo por citar unos ejemplos.
} 
urbanos marginados pierden sentido en este contexto; la lucha pasa de un carácter revolucionario a un plano electoral. El segundo inciso privilegia nuevos mecanismos de resolución de conflictos, básicamente se pasa de la confrontación pública a la negociación privada, y el más claro ejemplo son los programas de combate a la pobreza, los cuales terminan convirtiéndose en programas corporativizados para los sectores sociales excluidos dentro de los procesos de la economía de mercado.

Por lo anterior, los movimientos sociales pierden terreno como eje explicativo de los procesos de cambio político y en su lugar aparecen nuevas formas de expresión del asociacionismo civil, sobre todo de las clases medias urbanas. Esto origina una gran confusión entre los analistas, ya que las formas que adquiere la organización social son menos visibles y claras, así, las ONG's, las asociaciones civiles, se convierten en una parte recurrente del nuevo entorno de la participación ciudadana.

Así, estas nuevas figuras de asociacionismo civil, responden a la insuficiencia del Estado para poder dar solución al nuevo tipo de demandas ciudadanas. Las organizaciones civiles se definen como "asociaciones libres de ciudadanos cuyo fin es actuar conjuntamente en el espacio público para contribuir a la resolución de problemas de la sociedad y llenar los vacíos de atención a las necesidades de la población dejados por la acción del Estado y del mercado”. ${ }^{11}$

Estas formas de asociacionismo son variadas e incluyen amplios espectros de la vida social; van desde grupos de autoayuda o mutualista, hasta agrupaciones deportivas y de carácter transitoria, en este sentido "las distintas formas de asocianismo son un reflejo de los diferentes espacios y formas de acción colectiva, que van desde el privatismo y el particularismo, hasta la acción pública con fines culturales". ${ }^{12}$ Estas agrupaciones se convierten en los nuevos puntos de referencia para entender a los nuevos movimientos sociales, ya que cuestionan los viejos patrones de acción política y la centralización del poder, de tal manera que, estamos ante movimientos culturales que retan los códigos tradicionales de la política en México.

Estos nuevos movimientos sociales adquieren fuerza a mediados de los ochenta, y tienen un impacto trascendental en la construcción de la agenda pública del Estado. Estas organizaciones demuestran la fuerza de sectores ilustrados de la clase media mexicana, quienes rescatan los valores que en países desarrollados se llaman postmateriales, y que son parte del discurso de estos

11 OLVERA RIVERA, Alberto J. (2000): "Organizaciones de la sociedad civil: breve marco teórico", en Documento de discusión sobre el Tercer Sector, 8. El Colegio Mexiquense. p. 13.

${ }^{12}$ Ibidem. p. 14 
nuevos movimientos. ${ }^{13}$ La característica básica de estos nuevos movimientos sociales podemos definirla a partir de que no buscan la confrontación directa con el Estado sino piden transformaciones en leyes o en políticas públicas.

Aunque dichas organizaciones no están exentas a una presencia de distintas corrientes ideológicas, políticas o religiosas tanto en su composición interna como en su discurso hacia el exterior; entonces, estas nuevas formas que construyen la composición de los nuevos movimientos sociales en México agrupa a sectores de izquierda radicalizada, de derecha conservadora, gente con recursos económicos, intelectuales, así como también a gente sin estudios y con niveles altos de pobreza-, es un espectro variado y diverso que pretende evidenciar la alta complejidad de la sociedad contemporánea.

Estos movimientos tienen dos características, son particularistas y universalistas: (a) particularistas porque han llegado a ser estructuras muy cerradas con vida propia y autónoma en relación a los subsistemas de la estructura y (b) universalistas ya que contienen una orientación cultural que cuestionan a la sociedad en general, así también, afectan a ciertas estructuras parciales del Estado.

Así, estas nuevas formas de organización social construyen movimientos sociales de carácter cultural que tratan de intervenir en la definición e implementación de las políticas públicas, así, las agrupaciones que conforman las acciones colectivas son diversas, con intereses muchas veces encontrados, sin embargo, lo que los une es que todas compiten en la arena político cultural, buscando reformas en los diversos aspectos de la vida social y sobre todo en los aspectos de la transformación de las prácticas políticas del ciudadano, buscando generar un cambio en la cultura política.

Cuando México entra formalmente a los circuitos de la globalización en $1986,{ }^{14}$ los criterios de modernización enunciados bajo los lineamientos neoliberales afectan a los actores sociales propios de nuestra modernidad política. La globalización destruyó los componentes fundamentales de la sociedad civil mexicana, los espacios de intermediación tradicional entre la sociedad y el Estado tendieron a desaparecer, así, sindicatos, partidos políticos y movimientos sociales sufrieron un proceso de reconfiguración en su concepción y en su acción.

En este sentido la firma del Tratado de Libre Comercio con los Estados Unidos y su entrada en enero de 1994 se convirtió en un "disolvente poderosísimo de lo social: de las identidades colectivas y los espacios de

\footnotetext{
${ }^{13} \mathrm{Y}$ que conforman a los grupos ecologistas, movimientos lésbico gays, grupos en defensa de los animales, etcétera.

${ }^{14}$ Se toma como referencia la entrada de México al GATT a mediados de los años ochenta del siglo pasado.
} 
interacción comunicativa y de formación crítica de lo público" ${ }^{15}$ ya que este proceso ha acarreado miseria, desintegración social, altos índices de marginación y baja calidad de vida. Es inconcebible pensar en un orden democrático político si no hay un orden democrático social.

Las fuentes de desintegración social se ubican en: (a) el estancamiento económico de los años ochenta del siglo pasado, (b) la mala distribución de la riqueza y la falta de creación de empleos, lo que genera (c) niveles insospechados de pobreza, por lo que la anomia y la desintegración social propia del período de crisis económica causó que el país entrara en una crisis societal y política. Esto obligó a que fuera necesario el establecimiento de nuevos criterios de unidad y cohesión en términos sociales. Nuevos liderazgos tomaron el control de la intermediación sociedad - Estado, de tal suerte que aparecieron nuevos temas en la agenda de la sociedad civil, por ejemplo: la recuperación del pasado indígena del país, los derechos negados a las mujeres y los homosexuales, la fijación de criterios de equilibrio entre la actividad económica y el medio ambiente.

Lo anterior quiere decir que este proceso de desintegración social permitió que se buscaran nuevos puntos de referencia social para poder cohesionarse, el parámetro de la distribución de la riqueza desaparece en el discurso de los movimientos sociales y aparecen los temas de la identidad, los derechos culturales y simbólicos de las minorías.

Así, el proceso de quiebre de la modernidad "occidentalizada" mexicana permitió la aparición de problemas que ancestralmente se habían negado o relegado a nivel social. Por lo que movimientos como el EZLN, ambientalistas o de género, tienen referencia y espacio en el nuevo entorno social del país, dando paso así, al estudio de los movimientos sociales desde la perspectiva de las identidades.

\section{Los enfoques identitarios dentro del estudio de los movimientos sociales}

Las teorías de los movimientos sociales desde la perspectiva identitaria podemos dividirla en dos escuelas: un enfoque clásico y otro contemporáneo. Ambos corresponden al desarrollo del momento histórico de la evolución de las sociedades post industriales, sin embargo generan un cuadro heurístico general para el estudio de los movimientos sociales post materiales en el contexto social globalizado posteriormente informatizado.

\footnotetext{
${ }^{15}$ ZERMEÑO, Sergio. (1996): La sociedad derrotada: El desorden mexicano del fin de siglo, México Siglo Veintiuno Editores. p. 12
} 


\section{Enfoque clásico}

Los enfoques basados en la identidad parten del hecho de que los movimientos sociales son producto de la crisis de las sociedades postcapitalistas. El primer autor que maneja esta visión es el sociólogo francés Alain Touraine. Este autor parte de la crítica a la individualización extrema de la sociedad generada por la cultura del consumo del estado benefactor. Sus planteamientos inician las explicaciones de la aparición de los llamados nuevos movimientos sociales.

La estructura social formada por el modelo de Estado benefactor genera una serie de tensiones sociales que fragmenta a la sociedad. El corporativismo político y económico, la sociedad de masas, el ascenso de las clases medias, entre otras, fueron las condiciones generadoras de la estructura de los nuevos movimientos sociales. La fundación de estas nuevas agrupaciones sociales se entienden a partir de las acciones colectivas que tratan de recoger la diversidad de identidades generadas diferencialmente por el consumo y la democracia política. ${ }^{16}$

Así, el entorno social se vuelve diferenciado y fragmentado, forja una serie de problemáticas que superaban las visiones económicas y de clase, y se concentraban en posiciones simbólicas y de calidad de vida. La solución para estos problemas no se encontraba en posiciones políticas maximalistas, por esto se abre una fisura en el sistema político en dos sentidos: por un lado, (a) movimientos que luchan por el medio ambiente, los derechos humanos e igualdad de género, se empiezan a plantear en función de problemas civilizatorios propiciados por un sistema capitalista que se preocupaba más por su preservación que por las sociedades humanas y, por el otro lado, (b) un agotamiento de los canales de representación política tradicional, ya que los partidos políticos, el parlamento, los canales corporativistas de intermediación entran en una crisis de legitimidad.

La aparición de nuevo actores sociales que tratan de resolver esta crisis de legitimidad política y de sentido en la estructura social permite la aparición de estos nuevos movimientos sociales. Así los movimientos verdes, los movimientos étnicos, de género, etcétera, se convierten en los referentes inmediatos para entender la crisis del modelo capitalista de los años sesenta y setenta. Estos movimientos se presentaron de manera distinta en los diferentes países del mundo: mientras que en Europa y Norteamérica se presentan como luchas por derechos de género y civiles, en México se presentan de forma de luchas prodemocráticas, tales como el movimiento estudiantil del 68 o las movilizaciones en defensa del voto en los primeros años de la década de 1980.

${ }^{16}$ ALONSO, Luis Enrique. (1994): Crisis y transformación de los movimientos sociales en un entorno posffordista, en Comportamiento politico y electoral. DEL CASTILLO, Pilar. Centro de Investigaciones Sociológicas. Madrid. 
En esta lógica, Touraine define a los movimientos sociales como "interacciones normativamente orientadas entre adversarios con interpretaciones en conflicto y modelos societales opuestos, en un campo cultural compartido", ${ }^{17}$ es decir, este autor pone en el centro del debate la cuestión de la generación de las identidades colectivas como motor de las acciones sociales y por lo tanto, del movimiento en sí. Sin embargo, el punto de engarce con la transformación social está en el hecho del que estos movimientos llegan a reconocerse como portadores de un proyecto histórico distinto para la sociedad.

Touraine en su libro La producción de la sociedad(1995), da su versión más acabada de su teoría acerca del reconocimiento del sentido histórico de los movimientos sociales, comenta que este tipo de acción social surge debido a la tensión que se vive entre los planteamientos de un mundo capitalista tecnocrático, construido sobre la idea de la importancia de los objetos materiales respecto a un mundo simbólico del disfrute y la estética, las cuales son dos posiciones encontradas acerca del consumo. En esta lógica, la confrontación entre estos dos grupos con visiones en conflicto se convierte en el origen de los movimientos sociales.

El principio del reconocimiento de la presencia de un enemigo del proyecto social vigente permite abordar el problema de la naturaleza de los movimientos sociales que puede ubicarse en tres extremos interconectados entre sí: identidad, oposición y totalidad; (a) identidad se refiere a la definición del actor en sî mismo, el principio aglutinador de grupos sociales por la afectación de un problema determinado, (b) oposición en relación a que el conflicto determina a un adversario, el cual representa al enemigo, y por lo tanto, refuerza el principio de la identidad y (c) totalidad, que hace referencia al proyecto social en su conjunto, que es donde el movimiento social aporta el germen del cambio en términos sociales; comenta que la totalidad es "el sistema de acción histórica" en donde los adversarios se disputan el poder y el control social.

Touraine propone que la totalidad acarrea el proyecto de transformación social ya que la visión holística de la totalidad permite el ejercicio de la autorreflexión de la sociedad, por lo que la problematización de la estructura social permite visualizar que la sociedad postcapitalista se encuentra en peligro debido a los daños medioambientales del progreso económico e industrial o el reconocimiento de derechos negados a comunidades amplias llámese indígenas u homosexuales, por lo que la humanidad se encuentra atrapada en la lógica de la racionalidad instrumental.

${ }^{17}$ COHEN, Jean L. (1985): Strategy or Identity: New Theoretical Paradigms and Contemporany Social Movement. Social Research. 52: 663 - 716. 
Este autor hace aportaciones muy importantes a la teoría de los movimientos sociales, vincula la estrategia con la identidad como parte de los principios de totalidad, reconoce que los movimientos construyen su lucha alrededor de los patrones culturales que cohesionan y dan sentido a las sociedades actuales, por lo que la concepción de la autoorganización social será el de un instrumento de cambio de los sistemas sociales.

En esta misma lógica de diferenciar a los movimientos a partir de las crisis sistémicas del capitalismo, tenemos al autor alemán Claus Offe (1988), quien parte de entender la participación social desde la construcción de lo que él llamó el "viejo" y "nuevo" paradigma, con este concepto hace una distinción entre los viejos movimientos sociales de clase, donde se respondía de acuerdo a los criterios de distribución de los bienes materiales, mientras que el nuevo paradigma hace referencia a los sectores diferenciados de la sociedad postindustrial, los cuales tienen valores postmateriales, ${ }^{18}$ los cuales son valores universales y no tiene referencia específica a ninguna clase social, así que temas como ecologismo, feminismo, entre otros, son temas incorporados por estos nuevos movimientos sociales.

En este sentido, el tipo de demandas corresponde a momentos históricos específicos, comenta que el viejo paradigma se correspondería con una estructura social compuesta de colectividades relativamente duraderas y relativamente diferenciadas, tales como clases, agrupaciones según el status social, profesión, interés económico, comunidades culturales y familias. Por otro lado, el nuevo paradigma se estructura a partir de un grado más alto de individuación y diferenciación, es decir, a un tipo de estructura social en el que tales colectividades se han vuelto a la vez menos diferenciadoras y menos duraderas como puntos de referencia orientativos.

A diferencia de Touraine, sí toma en cuenta a la sociedad civil, tanto como elemento activo en la composición de los movimientos como en la reconfiguración de las relaciones entre el Estado-sociedad.

La crisis sistémica no sólo trae problemas administrativos, sino también de legitimidad y legalidad en los sistemas políticos, así que el reconocimiento de los derechos de autodeterminación contenidos en los movimientos sociales llevan la lógica de restituir los lazos entre la cada vez compleja y demandante sociedad civil; y el debilitado Estado rebasado en su capacidad de dar solución a demandas específicas. Offe dice que "en la sociedad moderna el problema central de la política democrática es mantener la diversidad en el interior de la sociedad civil, tratando de conseguir en cierta medida unidad, o 'ligazón' desde la autoridad política (1988: 168).

18 Sobre este tema, se puede revisar a Inglehart (1977). 
Una de las vertientes más interesantes del estudio de los movimientos sociales es el de las identidades, ya que parte de la necesidad de entender los procesos de aprendizaje al interior como al exterior de los movimientos.

\section{Enfoques contemporáneos}

Los enfoques de las identidades tienen una referencia en la democratización de la vida cotidiana, estas teorías parten de la perspectiva de que los movimientos sociales no sólo crean un compromiso de cambio estructural en la política sino que implican procesos de aprendizaje por parte de la sociedad civil (a partir del reconocimiento de la capacidad de autorreflexión y autoorganización) que aplican en su vida diaria.

Así, el establecimiento de los derechos en términos efectivos, los mecanismos de rendición de cuentas del poder público, el reconocimiento de la ciudadanía diferenciada y el establecimiento de los mecanismos de comunicación entre la sociedad y las esferas de poder van a adquirir materialidad en los movimientos sociales.

El argumento central de estas teorías se basa en la idea de que la sociedad ha transitado de una etapa industrial a la "sociedad de la información". Esta transformación tiene su origen en el hecho de que se dio una "autonomización de las esferas societales y sistémicas que en la modernidad industrial permanecían aún altamente indiferenciadas". ${ }^{19}$ La creación de los espacios públicos producto de la revolución informática posibilitó el acceso inmediato a los mecanismos de toma de decisiones sistémicas, además los procesos de creación de identidades generó nuevos referentes -ya no la clase social o la adscripción a un status social-determinados por valores particulares o postmateriales.

Los ciudadanos parten entonces de un estado de identidades fragmentadas, ya sea por la complejidad de las sociedades modernas o por el efecto de los medios de comunicación, por lo que los ciudadanos al reunirse en los movimientos sociales construyen una serie de redes sociales que crean identidades grupales o colectivas, por lo que estos espacios generan una lógica comunitaria muchas veces universalista (medio ambiente, derechos humanos) y, por otro lado, tienen sentidos restringidos en sus formas de lucha (pedir la modificación a una ley ambiental o evitar la construcción de un aeropuerto o carretera).

${ }^{19}$ ISUNZA Vera, Ernesto. (2001): Las tramas del alba. Una visión de las luchas por el reconocimiento en el México contemporáneo (1968 - 1993). CIESAS - Miguel Ángel Porrúa, México. 
Melucci argumenta en este sentido que estas redes sociales tienen muchas veces la finalidad de elaborar significados alternativos a los dominantes. Esta lógica incluye una concepción de revolución de los significados, acercándose a las teorías interpretativas y alejándose de los planteamientos racionalistas de las movilizaciones sociales.

Los códigos sociales pueden ser quebrados mostrando las incoherencias entre el lenguaje y las acciones estatales - un discurso medioambientalista pero una acción que rompa el equilibrio natural de una bio región, por citar un ejemplo-, en este sentido ${ }^{20}$ nos comenta que "en su propia actividad constitutiva y en su misma forma de presentar las internas relaciones de poder, los movimientos sociales pueden también subvertir los códigos dominantes: Esto es, demostrando que, al hacer visible el poder, no sólo se lo anula sino que se lo controla".

Entonces, el principio de acción y autolimitación está sujeto no sólo a las condiciones estructurales del movimiento, sino también a esta negociación de sentido al interior del mismo; y con respecto a los códigos afectados en la negociación con el sistema en general. Y es en este sentido que toma vigencia el concepto enunciado por Eyerman y Jamison, quienes construyen una visión cognitiva para abordar a los movimientos sociales.

Los movimientos sociales pueden concebirse como "manifestaciones de redes socioespaciales latentes, cuyo elemento aglutinador son sobre todo comunidades de valores. ${ }^{21}$ Estas comunidades de valor se construyen a partir de la interacción social, por lo que la población genera sus propios sentidos de la interacción entre el sistema y la sociedad. Es en esta dinámica, donde se generan los conocimientos necesarios para determinar si la sociedad se organiza o no, y bajo qué mecanismos van a organizar su acción.

\section{(a) Enfoque cognitivo}

El enfoque cognitivo define a los movimientos sociales "como formas de praxis cognitiva las cuales están conformadas por procesos políticos internos y externos. Los movimientos sociales expresan cambios en la conciencia de los actores a partir de la manera en que están articulados en las interacciones entre los activistas y sus opositores en un contexto político y cultural determinado" (Eyerman y Jamison, 1991: 4). Así, las personas crean nuevas formas de identidad a partir de la praxis cognitiva. Este concepto significa la identidad más profunda del movimiento: son los parámetros que establecen los miembros del grupo, las demandas, pero también son los criterios de identidad individual como miembros

\footnotetext{
${ }^{20}$ ISUNZA Vera, Ernesto. (2001): Las tramas del alba. Una visión de las luchas por el reconocimiento en el México contemporáneo (1968 - 1993). CIESAS - Miguel Ángel Porrúa, México. p. 100

21 DALTON, Russell, KUCHLER, Manfred (Compilador). (1999): Los Nuevos Movimientos Sociales: un reto al orden político, España, Editorial Alfonso Magnanim. p. 98
} 
del movimiento; así también, genera una identidad grupal que identifica al movimiento en relación al otro y le da legitimidad a sí mismo.

El enfoque cognitivo utiliza la metodología de los enmarcamientos (Frames) elaborada por Goffmann, y modificada para su utilización en el tema de los movimientos sociales por Johnston, Rivas y Eyerman y Jamison. Esta metodología reconstruye los elementos discursivos y la práctica al interior de los movimientos sociales, así como los referentes empíricos que generan la identidad individual y grupal de los mismos; esta teoría estudia la participación de los individuos dentro de los movimientos sociales para ver las transformaciones de la vida privada en relación a las peticiones de transformaciones del espacio público.

Podemos definir y caracterizar a los movimientos sociales desde una perspectiva cognitiva a partir de los siguientes elementos: es una red de ciudadanos que se organizan a partir de (a) un principio de diferenciación en relación a las esferas que componen la estructura social, (b) un carácter autolimitado, en relación a la distinción de los campos de referencia para su lucha, las cuales incluyen las autoreferenciales de los mismos: (1) un principio de identidad en relación y con respecto a sus demandas, (2) un autoaprendizaje a partir de sus demandas y (3) aceptan la existencia de las esferas del estado y el mercado capitalista, a partir de reconocer que estas esferas van a ser los principales blancos de sus demandas de transformación. ${ }^{22}$

En resumen, esto quiere decir que los nuevos movimientos sociales tienen la característica principal de "interpretar sus acciones como un intento de reformular la cultura política democrática y reintroducir la dimensión normativa de la acción social dentro de la vida política. Esto sería el significado del radicalismo autolimitado". ${ }^{23}$

\section{(b) Enmarcamientos discursivos en los movimientos sociales}

A partir de lo anterior, el estudio de los movimientos sociales desde una perspectiva contemporánea utiliza muchas aristas analíticas, pero las que puede ayudarnos a entender de una manera más integral el problema de la construcción de valores y prácticas democráticas dentro de contextos sociales post autoritarios sería la perspectiva cognitiva, ya que su análisis incluiría el problema de las transformaciones de las percepciones sociales en cuanto a la participación política, y el potencial democratizador o la refuncionalización de las prácticas autoritarias dentro de los imaginarios políticos de la sociedad.

En este sentido, el análisis de la perspectiva cognitiva se operativiza a partir de los enmarcamientos (también conocidos como "marcos" oframes) generados dentro de las prácticas discursivas al interior de los movimientos sociales. Estos

${ }^{22}$ COHEN, Jean L. (1985): Strategy or Identity: New Theoretical Paradigms

and Contemporany Social Movement. Social Research.

${ }^{23}$ Ibídem, p. 670. 
enmarcamientos parten del concepto de la autorreflexibilidad. La autorreflexibilidad es una parte importante de la construcción de las identidades en los movimientos sociales, ya que "los movimientos tienen sentido para los que participan en sus actividades, pero también pueden tenerlos para los que llegan a conocerlos a través de diferentes procesos de comunicación".

Con base a esta propuesta de análisis, entiendo un movimiento social como una construcción compleja que rebasa las explicaciones tanto racionalistas como simbólicas, de tal manera que los enmarcamientos nos permiten vincular las causas estructurales propias de los movimientos con las prácticas simbólicas de los mismos.

Las representaciones o interpretaciones que generan los movimientos sociales son el producto de una sumatoria de acontecimientos diversos que deben dilucidarse como resultado y no como punto de partida de ciertos procesos sociales. En este sentido, "la tarea del analista de los movimientos sociales consiste en explicar cómo ese resultado se construye colectivamente, cómo se mantiene y cómo podría cambiar con el tiempo". En otras palabras, comprendiendo cómo se construye el discurso y las acciones de los movimientos, se entenderá el potencial democratizador o no de la acción social colectiva.

Así, la perspectiva de los marcos (Frames), permite entender la manera en que los individuos construyen sus referentes para las acciones colectivas e individuales. Rivas nos dice en este sentido que "por 'procesos de enmarcamiento' entendemos los esfuerzos estratégicos conscientes realizados por grupos de personas para construir interpretaciones compartidas del mundo y de sí mismos que legitiman y motivan la acción colectiva"; sin embargo, yo agregaría, que los marcos no sólo se construyen por la cultura o la ideología, sino también por las formas estructurales organizativas del propio movimiento. ${ }^{24}$

Esta visión nos abre la posibilidad de incorporar nuevos caminos para el entendimiento de las acciones colectivas en un contexto post autoritario. Los marcos nos enseñan las maneras en que se construye el discurso, mientras que las formas de la acción pueden enseñar la intencionalidad pragmática de la acción política de los miembros del movimiento. Así mismo, los marcos sirven como una herramienta para la obtención de datos para la investigación acerca de movimientos sociales. Una de las propuestas más acabadas acerca de los enmarcamientos, no las da Rivas en su artículo "El análisis de marcos: Una metodología para el estudio de los movimientos sociales" (Rivas, 1998: 181-215) en el cual, construye un tipo ideal acerca de los marcos, a partir de enumerar dimensiones y estrategias del enmarcamiento en casos específicos de estudios de movimientos sociales.

${ }^{24}$ Recuperando así las escuelas de corte estructuralista de los movimientos sociales (Movilización de Recursos, Estructura de Oportunidades Políticas), las cuales como ya se explicó, fueron poco utilizadas en México, y con ello, se perdió una veta de análisis sobre los movimientos sociales muy importante. 


\begin{tabular}{|c|c|}
\hline $\begin{array}{l}\text { 1. Los indicadores construidos por } \\
\text { Rivas quedarían de la siguiente manera } \\
\text { Dimensiones o áreas temáticas }\end{array}$ & $\begin{array}{l}\text { Estrategias del enmarcamiento (o } \\
\text { técnicas para interpretar las áreas } \\
\text { temáticas) enmarcamiento. }\end{array}$ \\
\hline $\begin{array}{l}\text { 1) El movimiento indica una cuestión } \\
\text { de debate público. } \\
\text { 2) La cuestión del debate público debe } \\
\text { definirse como un problema } \\
\text { resaltando la discrepancia ser- deber } \\
\text { ser. } \\
\text { 3) Definición de la causa del problema. } \\
\text { 4) Identificación de los agentes } \\
\text { involucrados en el problema. } \\
\text { 5) Enmarcamiento de los objetivos. } \\
\text { 6) Enmarcamiento de las posibilidades } \\
\text { de éxito para la resolución del } \\
\text { problema. } \\
\text { 7) Enmarcamiento de los destinatarios } \\
\text { de la propuesta. } \\
\text { 8) Autolegitimación de los movimientos } \\
\text { sociales. }\end{array}$ & $\begin{array}{l}\text { 1) Asignación de un concepto o } \\
\text { eslogan de la lucha del movimiento. } \\
\text { 2) Contrastarlo empíricamente y } \\
\text { situarlo en sujetos concretos que } \\
\text { se encuentran fuera del } \\
\text { movimiento. } \\
\text { 3) Moralizar los temas, } \\
\text { esquematizarlos con valores o } \\
\text { dramatizar sus implicaciones para } \\
\text { el futuro. } \\
\text { 4) Darle credibilidad al movimiento. }\end{array}$ \\
\hline
\end{tabular}

Elaborado según Rivas (1998)

Respecto a esta tabla de dos columnas de indicadores metodológicos agregaría una tercera para analizar el contexto social o incluso personal de cada uno de los miembros o líderes de los movimientos sociales, ya que las biografias personales o colectivas no se ha explotado de manera clara como fuente de análisis del estudio del comportamiento y de las trayectorias políticas de la acción colectiva social.

2. Propuesta de indicadores para medir la dimensión personal - ideológica de los participantes de un movimiento social o un líder de un movimiento social

\begin{tabular}{|l|l|}
\hline $\begin{array}{l}\text { Dimensiones o áreas temáticas del } \\
\text { enmarcamiento. }\end{array}$ & $\begin{array}{l}\text { Estrategias del enmarcamiento (o } \\
\text { técnicas para interpretar las áreas } \\
\text { temáticas). }\end{array}$ \\
\hline $\begin{array}{l}\text { Análisis de la dimensión personal- } \\
\text { ideológica de los participantes del } \\
\text { movimiento. }\end{array}$ & $\begin{array}{l}\text { 1) El análisis de la personalidad del } \\
\text { dirigente del movimiento 2) la } \\
\text { ubicación del pasado político del líder } \\
\text { y los miembros entrevistados 3) el } \\
\text { establecimiento de relaciones directas } \\
\text { o indirectas con otros movimientos } \\
\text { sociales. }\end{array}$ \\
\hline
\end{tabular}

Cuadro elaborado por Luis Fernando Villafuerte Valdés 
En este sentido, metodológicamente, el empleo de los enmarcamientos obliga a la utilización de una perspectiva metodológica cualitativa, en la que las fuentes orales juegan un papel muy importante, ya que el empleo de estas técnicas permite visualizar la manera en que los miembros de los movimientos sociales construyen sus referencias simbólicas de la acción colectiva

Así, el estudio de los movimientos sociales desde la perspectiva cualitativa y con el empleo de las fuentes orales, permite analizar la realidad del movimiento desde tres ángulos: “(a) los procesos objetivos, (b) las formas como las personas y los grupos perciben estos procesos, y (c) las experiencias que se tienen en torno a ellos". De tal forma que metodológicamente el uso de fuentes orales permite entender no sólo los marcos sino que enseña cómo es que se construyen, por lo que entonces la acción colectiva adquiere sentidos y significados.

Esta técnica no sólo implica involucrarse activamente en el movimiento sino que reconstruye y recrea valores, cosmovisión, gestas, íconos, e incluso, las redes simbólicas identitarias o discursivas que le dan sentido a la acción colectiva. Este esquema permite construir una mirada metodológica más estructurada al estudio de los movimientos sociales, ya que se le da una aproximación hermenéutica a los movimientos que se investigan, lo que origina que se pueda interpretar la lucha de los movimientos sociales más allá de los problemas visibles por los cuales se organizan, es decir, el impacto sobre la sociedad por parte de la acción colectiva puede ser fundamental para establecer por ejemplo; condiciones de creación de una cultura política democrática, o prácticas ciudadanas más complejas, sin embargo, este potencial está condicionado a que los dirigentes y los miembros del movimiento sean capaces de descifrar estos códigos "ocultos" inmersos en su propia lucha, generando así el sentido cognitivo enunciado por Eyerman y Jamison.

\section{CONCLUSIONES}

La relación del ambiente intelectual con el ámbito político en México fue muy evidente en los años en que imperaba el sistema político autoritario en el país. Las tradiciones que investigaban a los movimientos sociales fueron muy limitadas debido a dos razones: (a) un sistema político que orilló a que las experiencias empíricas de movilización social no tuvieran márgenes de maniobra para un desarrollo consolidado, por lo que el aniquilamiento o corporativización de los movimientos sociales no dejó dudas de la ausencia de autonomía de la sociedad civil con respecto al Estado, y (b) la única fuente de análisis teórica que tuvo un mayor eco en sus reflexiones fue la marxista, pero esta perspectiva restringió la idea del potencial de los movimientos sociales a una visión reduccionista de la participación política en términos de la arena política, dejando de lado el impacto de la movilización social en los referentes culturales e ideológicos en la sociedad. 
Podemos decir que en México, no existió una autonomía real entre los ámbitos académicos y las esferas políticas, por lo que la inexistencia de un espacio público consolidado, crítico y autónomo impactó de manera muy clara en la utilización ciertas teorías sociales para explicar los procesos políticos sociales en México.

En este sentido, la complejización de las relaciones sociales en México a partir de la disolución de los mecanismos tradicionales de control político sobre la sociedad; producto de las medidas neoliberales de finales del siglo XX, permitieron no solamente cambios en las dinámicas sociales, sino también en la forma en que se explicaban los fenómenos políticos sociales, es decir, el ambiente intelectual abandonó las visiones maximalistas como el eje analítico de las posibilidades de cambio social, pasando al entorno de la cultura como el lugar en donde los miembros de la sociedad pueden tejer sus alternativas (la dicotomía entre autoritarismo o democracia) para el establecimiento de prácticas ciudadanas más participativas o democráticas, o en su defecto, refuncionalizar las prácticas autoritarias en el país.

Así, las teorías cognitivas de los movimientos sociales se empezaron a utilizar en México en la última década del siglo pasado y su uso se propagó en la medida que era necesario estudiar si estos nuevos movimientos sociales postmateriales tienen la fuerza social suficiente para plantear un cambio significativo en el modelo político social en el país.

Para dar cuenta de este potencial, la utilización del enfoque cognitivo como propuesta analítica para el estudio de los nuevos movimientos sociales en México va acompañada del desarrollo metodológico de los enmarcamientos. Esta estrategia metodológica tiene como eje el estudiar si es que hay una correspondencia entre los discursos generados por parte de los miembros de un movimiento social con respecto a sus actitudes políticas; y así poder analizar su congruencia o incongruencia en cuanto a sus planteamientos políticos, ya que esto nos permitirá entender las dificultades que atraviesa la sociedad civil para su consolidación bajo los contextos post autoritarios.

Así, los enmarcamientos nos permitirá estudiar y narrar las experiencias regionales de lucha por la conformación y acción de la sociedad civil en cuanto a la participación social, ya que el sustento del cambio político esta en las transformaciones de las tradiciones políticas ejemplificadas en la cultura política, y para poder dar cuenta de estas transformaciones sociales en la cultura debemos acercarnos a sus marcos interpretativos de acción y a partir de ellos visualizar su percepción acerca de su papel en relación al proceso de cambio político regional, estatal y nacional.

De esta forma, esta metodología nos permite hacer una lectura no mitificada de los movimientos sociales, lo cual nos permita establecer el peso de las inercias 
de la tradición autoritaria en la sociedad civil, ya que gran parte del discurso de los teóricos de los movimientos sociales dan un tratamiento sobreideologizado acerca de la auto organización social, manejando una dicotomía simplista, el Estado que es el causante de los males políticos y la sociedad civil que es el instrumento de justicia e igualdad que nos va a ayudar a salvar a la sociedad.

Así, para entender a los actores dentro de los movimientos sociales, tenemos que partir del hecho que hay congruencias o incongruencias entre el discurso de la gente que pertenece a los movimientos, ya que tienen elementos autoritarios (el preservar figuras corporativas y de clase al interior del movimiento) y elementos democráticos (una lectura alternativa a los problemas sociales). En este sentido, esta perspectiva tiene una clara aproximación sociológica a los movimientos sociales, partiendo de elementos propios de la ciencia política, los cuales nos ayudan a reconstruir las redes y las tramas de los miembros de los movimientos y poder darle sentido así a estos discursos.

El potencial del análisis de los actores sociales nos permitirá ver las posibilidades que tenemos para construir sistemas sociales más justos y equitativos y más democráticos, ya que la democracia también se construye desde abajo, desde lo social, desde lo cotidiano, y el entender el cómo es que esos valores democráticos se establecen en los imaginarios sociales nos llevaría a construir los parámetros de una democracia de calidad, la cual nos llevaría a entender una vida digna con más calidad, y así poder decir que ya se ha establecido la democracia en México.

\section{FUENTES}

Archivo Histórico Municipal de Cazones de Herrera, Veracruz. Biblioteca Veracruzana, Colección de Publicaciones Periódicas El Diario de Xalapa, Diario de la Provincia, 20 Enero de 2007.

\section{SELECCIÓN BIBLIOGRÁFICA}

ALONSO, Luis Enrique. (1994): "Crisisy transformación de los movimientos sociales en un entorno postfordista”, en Pilar del Castillo. Comportamiento político y electoral. Centro de Investigaciones Sociológicas. Madrid.

AVRITZER, Leonardo. (2002): Democracy and the Public Space in Latin American. Princenton University Press y New Jersey.

BERGER, Peter y THOMAS Luckmann (1998): L a construcción social de la realidad. Amorrortu, Buenos Aires.

CASQUETTE, Jesús. (1998): Política, cultura y movimientos sociales. Bakeaz. Bilbao.

CASTREJÓN DIEZ, Jaime. (1995): La politica según los mexicanos. Océano, México.

COHEN, Jean L. (1986): Strategy or Identity: New Theoretical Paradigms and Contemporany Social Movement. SocialResearch. 52: 663- 716. 
DE LA GARZA TOLEDO, Enrique. (1988): Ascenso y crisis del Estados social autoritario. Estado y acumulación del capital en México (1940 - 1976). El colegio de México. México.

DE LA PORTA, Donatella. (1997): Social Movement. An Introduction. Temple University Press, Filadelfia.

DRESSER, Denisse. "SEDESOL. Los dilemas de la gobernabilidad”, en El Cotidiano. 1992. Núm. 49, Julio- Agosto, Universidad Autónoma Metropolitana - Azcapotzalco. Friederich Ebert Stiftung, México.

EYERMAN, Ron y ANDREW Jamison. (1991): Social Movement. A Cognitive Aproach. Pennsylvania State University, Pennsylvania.

GALINDO CÁCERES, Jesús. (1998): Técnicas de Investigación en estudios de sociedad, comunicación y cultura. Consejo Nacional para la Cultura y las Artes. México.

GOFFMAN, Erwin. (1997): La presentación de la persona en la vida cotidiana. Amorrortu, Buenos Aires.

HABERMAS Jürgen. (1998a): Problemas de legitimación en el capitalismo tardío. Amorrortu Editores, Buenos Aires.

HABERMAS, Jurgen. (1998b): Facticidezy V V alider. Sobre el derecho y el estado democrático de derecho en términos de teoría del discurso. Ed. Trotta. Madrid.

IBARRA, Pedro y Benjamín Tejerina.(eds). (1998): Los movimientos sociales. Transformaciones políticas y cambio cultural. Editorial Trotta. Madrid.

IBARRA Pedro. “QQué son los movimientos sociales?” En Anuario de los movimientos sociales, una mirada sobre la red. Elena Grau y Pedro Ibarra (Coord). 2000. Icaria Editorial y Getiko Fundazioa. Barcelona.

INGLEHART, Ronald. (1997): The Silent Revolution. Cambridge University Press, Inglaterra.

INGLEHART, Ronald. (1997): El cambio culturalen las sociedades avanzadas. Centro de Investigaciones Sociológicas, Madrid.

ISUNZA Vera, Ernesto. (2001): Las tramas del alba. Una visión de las luchas por el reconocimiento en elMéxico contemporáneo (1968 - 1993). CIESAS - Miguel Ângel Porrúa, México.

JOHNSTON, Hank y BERT Klandermans (eds). Social Movement and Culture. SocialMovements, Protest and Contention. 1995. Vol. 4. University of Minnesota Press, Minneapolis.

KEANE, John. (1997): “Transformaciones de la Esfera Pública”, en Estudios Sociológicos. Vol. XV, enero - abril. El Colegio de México, México. pp. 47 - 77.

VILLAFUERTE, Luis Fernando. (2008): “Una metodología interpretativa para el estudio de los movimientos sociales. Enmarcamientos y cultura. Una visión desde México", en Revista Historia de la Educación Latinoamericana $\mathrm{N}^{0} 11$, Tunja, Universidad Pedagógica y Tecnológica de Colombia, RUDECOLOMBIA, pp. 225-246 